DOI 10.17816/transsyst20184292-106

(C) D. Zhou ${ }^{1,2}$, P. Yu ${ }^{1}$, J. Li $^{1}$, P. Cui ${ }^{1}$, M. Song ${ }^{1}$

${ }^{1}$ National University of Defense Technology

(Changsha, China)

${ }^{2}$ State Key Laboratory of Functional Materials for Informatics

Shanghai Institute of Microsystem and Information Technology

Chinese Academy of Sciences

(Shanghai, China)

\title{
ADAPTIVE VIBRATION CONTROL OF THE ELECTROMAGNET- TRACK COUPLED HIGH FREQUENCY RESONANCE FOR AN URBAN MAGLEV SYSTEM
}

This paper is a study of the electromagnet-track coupled high frequency resonance that frequently occurs in the urban maglev systems, it includes the following points:

Aim: The purpose of this study is to investigate the principle underlying the high frequency resonance occurs between the maglev train and the track, and to develop an appropriate vibration control algorithm which can be applied in the levitation controller, such that the resonance can be eliminated when the maglev train travels along the track.

Methods: In this paper, the model of the electromagnet-track coupled system is firstly established, in which some special cases, which correspond to the situations when the screws that fasten the F-rail to the sleepers are fatigue, or the stiffness of the rubber plates beneath the sleepers weaken for temperature reasons, are studied; and the reason that leads to the coupled resonance are explained as well. Secondly, an adaptive vibration control algorithm, which consists of a vibration observer and a tunable adaptive filter, is designed to suppress the high frequency electromagnet-track coupled resonance.

Results: Using this algorithm, when the train arrives at the spots where the coupled resonance may occur, the vibration observer will detect the occurring of the vibration and estimates its frequency, and then activate the adaptive filter and tune it to absorb the vibration.

Conclusion: The test indicates that this algorithm is capable of tuning itself to handle the unpredictable coupled resonance that occurs along the track, and it is simple and can be easily integrated into the levitation control code in a digital levitation control system.

Keywords: Vibration, Resonance, Track, Control, Adaptive, Urban maglev.

\section{INTRODUCTION}

On December $30^{\text {th }}, 2017$, the Beijing S1 urban maglev line began its commercial operation after years of argument and construction ever since 2011. Earlier in May, 2016, the first low speed urban maglev line in China - the Changsha Maglev Express, began to operate. The successful operation of these two maglev lines in China, together with the commercial maglev lines in Japan and Korea, indicate that the low speed urban maglev technology is tending to be mature, and its spread around the world is predictable. 
However, as the development of test lines and the construction of commercial lines around China, more and more problems that related to the levitation control system of the train have been discovered. For example, the stability of the levitation control system under special track conditions, the reliability of the maglev controller, and the sensors under severe circumstances during the operation, etc. Another common problem for the urban maglev system may have been encountered - the vehicle-guideway coupled resonance (or coupled self-excited vibration in some literature). The coupled resonance occurs between the levitation control system and the track (or girder) when the track conditions are poor or the levitation control system is not well tuned. It is reported that the coupled resonance problem might be a fatal problem if it occurs on some light-weighted girders and cannot be well solved [1].

The maglev vehicle-guideway coupled resonance problem can be further divided into two major categories: the vehicle-girder coupled self-excited vibration, and the electromagnet-track coupled resonance. The former generally occurs on an elevated girder when the train is suspending in a standstill. It appears that the girder begins to vibrate and it can be clearly felt inside the train. The frequency of the girder vibration is generally below $30 \mathrm{~Hz}$, which is determined by the natural frequency of the girder, but the energy of the vibration is large enough to cause levitation failure or even damages to the system structures. These problem has been noticed and discussed by Zhou et al.[2] The electromagnet-track coupled resonance appears as the vibration of the steel rails when the electromagnet is suspending at some specified locations. It may appear not as severe as the vehicle-girder coupled self-excited vibration, but the frequency is generally above $50 \mathrm{~Hz}$, thus it can be clearly heard by passengers onboard the train or people inside the rooms beneath the tracks. To meet the requirements of the commercial line, such as the Beijing S1 maglev line, the electromagnet-track coupled high frequency resonance must be solved, and it is also the major problem to be discussed in this paper.

The electromagnet-track coupled resonance problem has also been noticed by Zhou et al., as discussed in [3-5], in which several methods to eliminate the coupled resonance has been tried. For example, in [3], an LMS cancellation algorithm has been proposed to generate a signal which is negative to the vibratory component mixed in the closed loop of the levitation control system, and different method for producing reference signals for the LMS algorithm have been proposed in these literature. In [5], the amplitude and frequency of the high frequency resonance can even be precisely computed, which agrees quite well to experiments. More detailed and comprehensive discusses of the coupled vibration problems can be found in the dissertation of Zhou [6]. In this paper, considering that the track conditions may differ as the train travels at different segments of the commercial line, and the amplitude, as well as the frequency, may vary as the train travels; therefore, a more reliable and robust method to eliminate the track-induced high frequency resonance needs to be developed. In 
this paper, the principle of the high frequency resonance is firstly introduced; and based on this theory, a new vibration control structure, which consists of a vibration observer and a tunable adaptive FIR (finite impulse response) filter, is designed to suppress the electromagnet-track coupled resonance. This adaptive structure can tune itself to cancel the vibration when the high frequency resonance tends to appear, and it is suitable to work while the train is traveling along the maglev lines.

\section{MODELING OF THE ELECTROMAGNET-TRACK COUPLED SYSTEM}

During the test runs of the maglev trains, it is discovered that the electromagnet-track coupled high frequency resonance always occurs at the locations where the screws which fasten the F-rail to the sleepers are fatigue or slack; another case occurs when the stiffness of the rubber plates beneath the sleepers are weaken for some reasons like temperature. Here, a model with a not well supported track is discussed, as shown in Fig. 1. The latter case mentioned above may also be characterized as a weakly supported track.

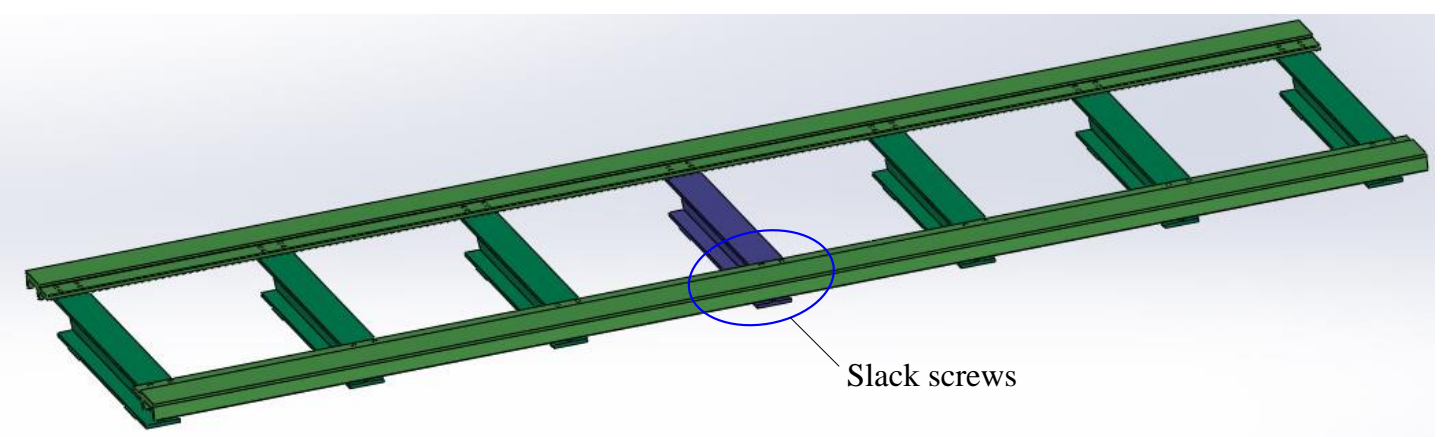

Fig. 1. A track model with slack screws connecting a sleeper and the rail

For the low speed maglev tracks, the rail is long and slim, thus it can be deemed as an Euler-Bernoulli beam with multiple supporters. A simplified model of the rail in each side of a track is shown in Fig. 2, in which each supporter is modeled as a combination of a linear spring and a torsal spring. The linear spring corresponds to the flexibility brought by the sleeper and the rubber plate beneath the sleeper; while the torsal spring characterizes the torsal rigidness of the sleeper since the rail are mounted to each sleeper by four hexagon socket screws. However, when the four screws are loose or fatigue, the stiffness of these springs will decrease or even disappear, leading to a weakly supported beam (say, the $k$-th support shown in Fig. 2). Fig. 2 also demonstrates the relative position of an electromagnet beneath the rail. 


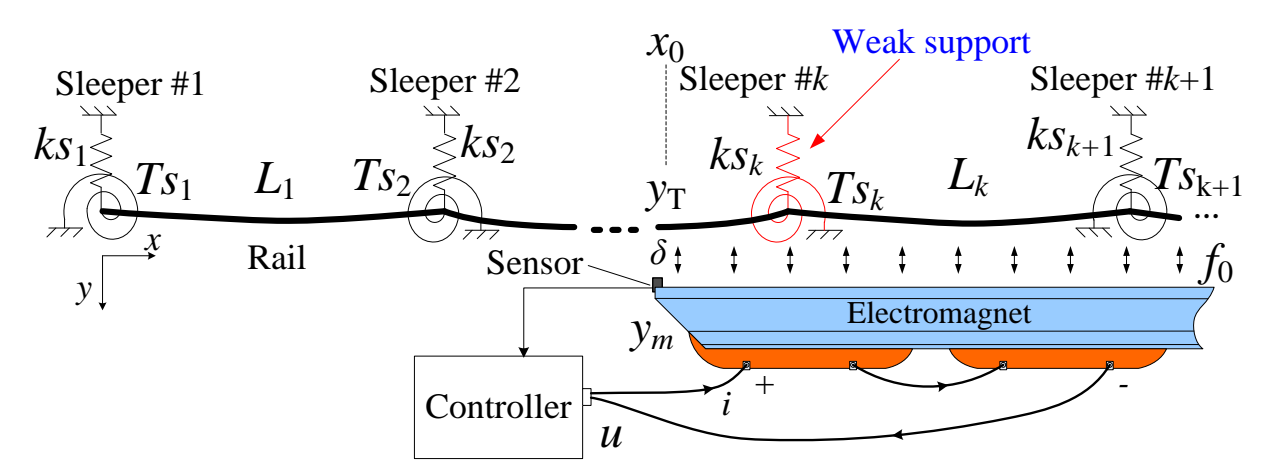

Fig. 2. Simplified model of the rail with multiple supporters

In Fig. 2, suppose that $E, I$, and $\rho$ are the Young's modulus of the track material, the moment of inertia of the track cross section, and the mass per unit length of the track, respectively; $k s_{n}$ and $T s_{n}$ represent the equivalent stiffness of these two springs at the $n$-th sleeper, respectively; and $L_{n}$ is the span length of the $n$-th span. Then, for the Euler-Bernoulli beam, the vertical displacement of the rail, $y(x, t)$, satisfies:

$$
E I \frac{\partial^{4} y(x, t)}{\partial x^{4}}+\rho \frac{\partial^{2} y(x, t)}{\partial t^{2}}=f(x, t)
$$

where $f(x, t)$ is the electromagnetic force distributed on the rail. Note that for the urban maglev system, $f(x, t)$ can be regarded as a evenly distributed force. Firstly consider the free vibration of the beam, and temporarily set $f(x, t)=0$. Suppose that the free vibration of the beam can be expressed as $y(x, t)=\phi(x) \sin \omega t$, then according to Eq.(1), we have

$$
\frac{E I}{\rho} \frac{\partial^{4} \phi(x)}{\partial x^{4}}-\omega^{2} \phi(x)=0
$$

Eq. (2) indicates that the form of the solution for the mode shape, $\phi(x)$, can be described as a combination of trigonometric and exponential functions, thus for the $n$-th span of the beam, it can be written as:

$$
\phi_{(n)}(x)=C_{n 1} \sin (\lambda x)+C_{n 2} \cos (\lambda x)+C_{n 3} \operatorname{sh}(\lambda x)+C_{n 4} \operatorname{ch}(\lambda x)
$$

where $\lambda$ is the spatial frequency. Substituting Eq. (3) into Eq. (2), yields:

$$
\omega^{2}=E I \lambda^{4} / \rho
$$

Referring to Fig. 2, it can be seen that for the $N$-th span beam, due to the effects of the springs, the bending moment and the shear force of the beam at the left terminal of the rail satisfy the following two equations, respectively:

$$
\begin{aligned}
& E I \frac{\partial^{2} \phi_{(1)}(0)}{\partial x^{2}}=T s_{1} \frac{\partial \phi_{(1)}(0)}{\partial x} \\
& E I \frac{\partial^{3} \phi_{(1)}(0)}{\partial x^{3}}=-k s_{1} \phi_{(1)}(0)
\end{aligned}
$$

For the intermediate supports, the balance equations for the bending moment and the shear force of the beam can be expressed as follows: 


$$
\begin{aligned}
& \left.E I \frac{\partial^{3} \phi_{(n)}(x)}{\partial x^{3}}\right|_{x=L_{n}}=\left.k s_{n+1} \phi_{(n)}(x)\right|_{x=L_{n}}+\left.E I \frac{\partial^{3} \phi_{(n+1)}(x)}{\partial x^{3}}\right|_{x=0} \\
& \left.E I \frac{\partial^{2} \phi_{(n)}(x)}{\partial x^{2}}\right|_{x=L_{n}}=-\left.T s_{n+1} \frac{\partial \phi_{(n+1)}(x)}{\partial x}\right|_{x=0}+\left.E I \frac{\partial^{2} \phi_{(n+1)}(x)}{\partial x^{2}}\right|_{x=0}
\end{aligned}
$$
satisfy:

At the right terminal, the bending moment and the shear force of the beam

$$
\begin{aligned}
& \left.E I \frac{\partial^{2} \phi_{(N)}(x)}{\partial x^{2}}\right|_{x=L_{N}}=-\left.T s_{N+1} \frac{\partial \phi_{(N)}(x)}{\partial x}\right|_{x=L_{N}} \\
& \left.E I \frac{\partial^{3} \phi_{(N)}(x)}{\partial x^{3}}\right|_{x=L_{N}}=\left.k s_{N+1} \phi_{(N)}(x)\right|_{x=L_{N}}
\end{aligned}
$$

Note that the continuities of the displacement and slope of the beam at the intermediate supports require

$$
\begin{aligned}
& \phi_{(n)}\left(L_{n}\right)=\phi_{(n+1)}(0) \\
& \phi_{(n)}^{\prime}\left(L_{n}\right)=\phi_{(n+1)}^{\prime}(0) .
\end{aligned}
$$

Combing Eqs. (5)-(12) together, there will be totally $4 \times N$ equations, and they can be written in a matrix form:

$$
\mathbf{A}_{\mathbf{m}} \mathbf{C}=0
$$

where $\mathbf{A}_{\mathbf{m}}$ is a $4 N \times 4 N$ matrix, and $\mathrm{C}$ is the coefficients of the mode shapes described by Eq. (3), namely, $\mathbf{C}=\left[C_{11}, C_{12}, C_{13}, \cdots, C_{N 3}, C_{N 4}\right]^{\mathrm{T}}$. By solving Eq. (13), the unknown coefficients, as well as the spatial frequency of the beam, $\lambda$, can be obtained.

The solution of Eq. (1) can be described as a combination of all the modal shapes obtained above, namely:

$$
y(x, t)=\sum_{k=1}^{\infty} q_{k}(t) \phi_{k}(x)
$$

Here, $y_{k}(t)$ is the generalized displacement of the $k$-th order mode. Substituting Eq. (14) into Eq. (1), multiplying both sides of the resultant equation by $\phi_{k}(x)$, and integrating both sides along the beam, noticing the mode orthogonality condition for linear beams [7], one obtains

$$
\ddot{q}_{k}(t)+\omega_{k}^{2} q_{k}(t)=\frac{F_{k}(t)}{M_{k}}
$$

where $M_{k}=\rho \int_{0}^{L} \phi_{k}^{2}(x) \mathrm{d} x, F_{k}(t)=\int_{0}^{L} f(x, t) \phi_{k}(x) \mathrm{d} x$, and $L=\sum_{k=1}^{N} L_{k}$.

Using the Laplace Transform, Eq. (15) can be rewritten as 


$$
q_{k}(s)=\frac{F_{k}(s)}{\left(s^{2}+\omega_{k}^{2}\right) M_{k}}
$$

Therefore, the vertical displacement of the beam at a specified location, $x_{0}$, can be given by

$$
y_{k}\left(x_{0}, s\right)=\frac{F_{k}(s) \phi_{k}\left(x_{0}\right)}{\left(s^{2}+\omega_{k}^{2}\right) M_{k}}
$$

For the electromagnetic force, $f(x, t)=F_{m}(t) / l_{0}$, where $F_{m}(t)$ is the total electromagnetic force produced by the coil pair, and $l_{0}$ is the length that the electromagnetic force covers. According to Fig. 2, it can be seen that

$$
F_{k}(s)=\frac{F_{m}(s)}{l_{0}} \begin{cases}\int_{x_{0}}^{x_{0}+l_{0}} \phi_{k}(x) \mathrm{d} x, & \text { if } x_{0}+l_{0} \leq L \\ \int_{x_{0}}^{L} \phi_{k}(x) \mathrm{d} x, \text { if } x_{0}+l_{0}>L\end{cases}
$$

Therefore, Eq.(17) can be rewritten as another form:

$$
y_{k}\left(x_{0}, s\right)=\frac{F_{m}(s) g_{k}\left(x_{0}\right)}{\left(s^{2}+\omega_{k}^{2}\right)}
$$

Here, $g_{k}\left(x_{0}\right)$ can be deemed as the $k$-th order mode gain of the beam, which is a coefficient that reflects the deformation response of the beam at $x_{0}$ when the beam is subjected to a unit force, $F_{m}$. The larger $g_{k}\left(x_{0}\right)$ is, the larger will be the displacement of the rail at $x_{0}$. As a demonstration, for the Beijing S1 low speed urban maglev system, $l_{0}=1.32 \mathrm{~m}, L_{n}=1.2 \mathrm{~m}, \rho=130 \mathrm{~kg} / \mathrm{m}, E I=$ $1.93 \times 10^{6} \mathrm{~N} \mathrm{~m}^{2}$ (the last two parameters of are obtained by calculation); the stiffness of the linear and torsal springs are supposed to be: $k s_{n}=1 \times 10^{8} \mathrm{~N} / \mathrm{m}$, and $T s_{n}=7 \times 10^{10} \mathrm{Nm} / \mathrm{rad}$. For the strongly supported rail, all the sleepers are well fastened to the rail, thus the parameters for all the springs are identical with each other. Using the procedure presented above, the first three order mode shapes can be computed, as shown in Fig. 3, and their corresponding mode frequencies are $122.2 \mathrm{~Hz}, 129.3 \mathrm{~Hz}$, and $141.5 \mathrm{~Hz}$, respectively. This result is rather close to finite element analysis. Meanwhile, the mode gains for these three vibration modes can be obtained as well, which are shown in Fig. 4.

As a comparison, consider the case when the screws of one sleeper slack. Specifically, suppose that the weak support appears at the middle sleeper (see Fig. 1). In this case, the stiffness of the linear spring and the torsal spring will be prominently weakened, which are supposed to be $1 / 10$ of the stiffness of other well fastened supports. The resultant vibration mode shapes as well as mode gains of the weakly supported rail can be computed, as shown in Fig. 5 and Fig. 6, respectively. Comparing Fig. 5 with Fig. 3, it can be seen that, due to the decrease of support stiffness in the middle of the rail, the shape of the first order mode shape changes dramatically - the displacement of the rail in the middle is more obvious than at other supports, which indicates that when the train moves 
here, the rail displacement will significantly increase. Also, Fig. 6 shows a prominent increase of mode gain at the middle for the first order vibration mode. According to Eq. (19), this suggests that the vertical response of the rail at the middle will be larger than at other locations when the train passes the rail. This is a fundamental reason that leads to electromagnet-track coupled resonance when the track support weakens.
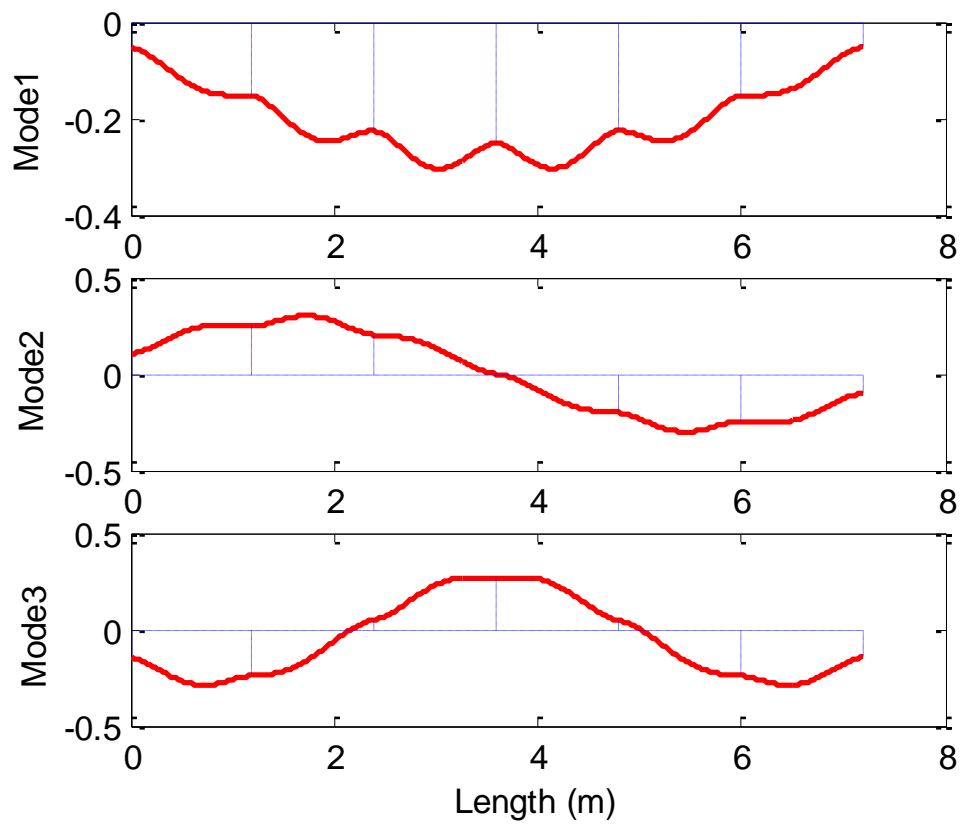

Fig. 3. The first three order mode shapes of the well-supported rail
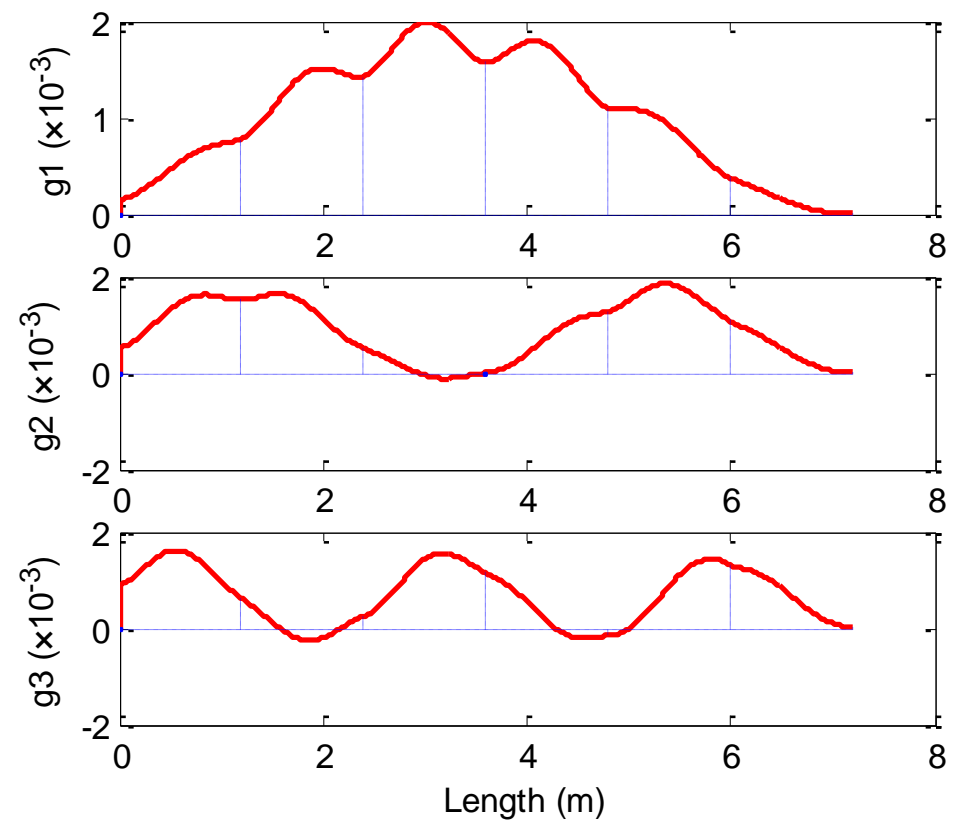

Fig. 4. Mode gains for the first three order vibration modes of the well-supported rail 

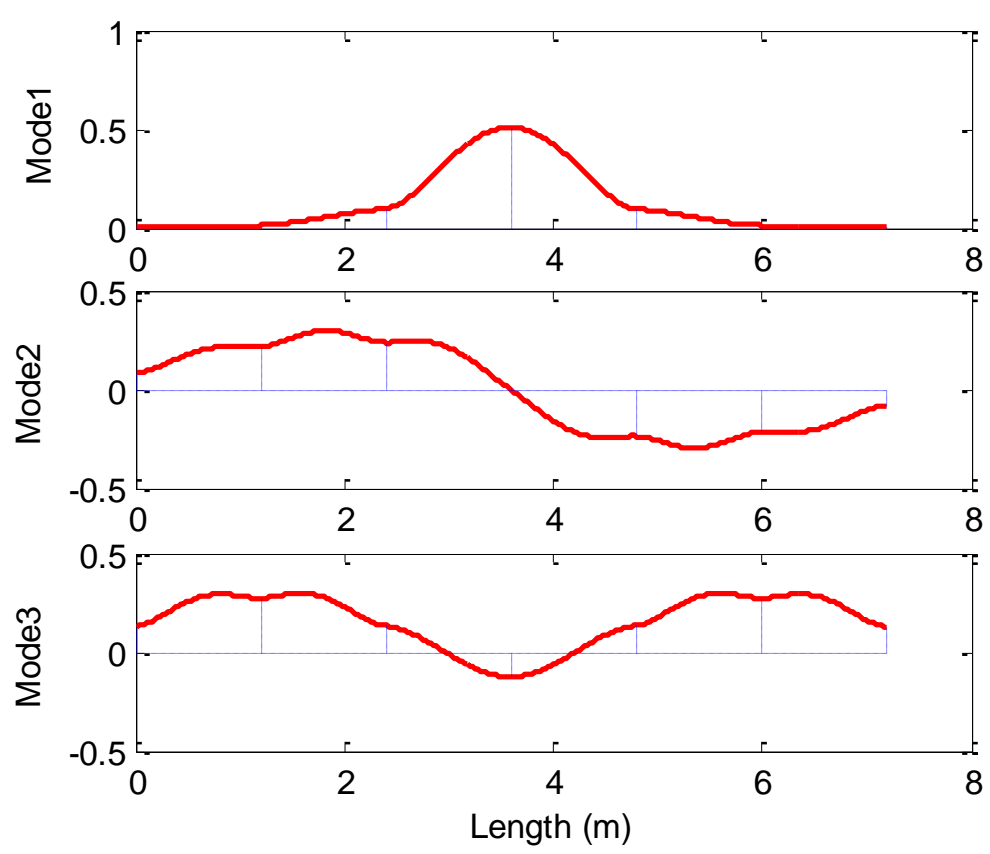

Fig. 5. The first three order mode shapes of the well-supported rail
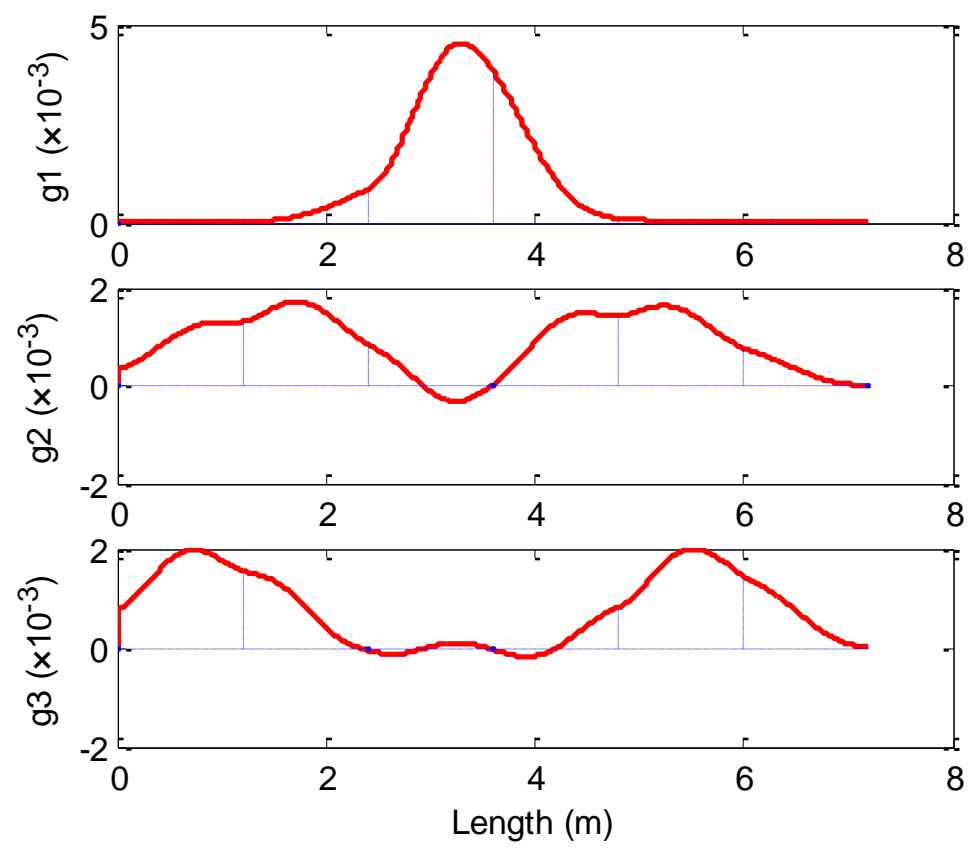

Fig. 6. The first three order mode shapes of the weakly-supported rail

Another significant change as a result of different rail support conditions is the mode frequency. For comparison, the mode frequencies, as well as mode gains when $x_{0}=3.3 \mathrm{~m}$, for these two cases are listed in Table 1 . It can be seen that the fundamental mode frequency drops significantly as the middle support becomes weak, while the other two higher mode frequencies only decrease a bit. This is another reason why the coupled resonance tends to occur when the rail is 
not well supported, since the lower the mode frequency is, the higher is the open loop gain, as is analyzed by Zhou et al. [2-6].

Table 1. Mode frequencies and mode gains for the normal and the weakly supported rail

\begin{tabular}{|c|c|c|c|c|}
\hline \multirow{2}{*}{ Mode order } & \multicolumn{2}{|c|}{ Mode frequency $(\mathbf{H z})$} & \multicolumn{2}{c|}{ Mode gain $\left(\times \mathbf{1 0}^{-\mathbf{3}}\right)$} \\
\cline { 2 - 5 } & Normal rail & Weak rail & Normal rail & Weak rail \\
\hline 1 & 122.2 & 76.4 & 1.82 & 4.53 \\
\hline 2 & 129.3 & 126.1 & -0.10 & -0.31 \\
\hline 3 & 141.5 & 133.2 & 1.51 & 0.10 \\
\hline
\end{tabular}

For the levitation control system shown in Fig. 2, the levitation gap $\delta$, which is the vertical distance between the electromagnet and the track measured by the gap sensor, can be described as

$$
\delta(t)=y_{m}(t)-y_{\mathrm{T}}(t)
$$

where $y_{m}$ is the vertical displacement of the electromagnet, $y_{T}$ is the vertical deformation of the track at $x_{0}$, which can be obtained by the superposition of all vibration modes using Eq. (14). To keep the levitation gap at a desired value, say, $z_{0}$, a controller is needed to eliminate the levitation gap error. The PID cascade controller, which has been employed in [8], is also adopted here, which gives

$$
i_{e}(t)=k_{p}\left[\delta(t)-z_{0}\right]+k_{d} \dot{y}_{m}(t)+k_{i} \int_{0}^{t}\left[\delta(\tau)-z_{0}\right] d \tau
$$

where $i_{e}$ is the desired current through the electromagnet coil. To achieve rapid response of the current, a current loop is utilized, and the control voltage is given by

$$
u(t)=k_{c}\left[i_{e}(t)-i(t)\right]
$$

Here, $k_{p}, k_{d}, k_{i}$, and $k_{c}$ are constants which can be chosen to achieve desired dynamic performance of the control system. The current through the electromagnet coil satisfies [3]:

$$
\frac{u(t)}{2}=i(t) R+\frac{\mu_{0} A N_{c}^{2}}{2 \delta(t)} \dot{i}(t)-\frac{\mu_{0} A N_{c}^{2} i(t)}{2 \delta^{2}(t)} \dot{\delta}(t)
$$

Here, $\mathrm{A}$ is the area of the electromagnetic pole, $\mu_{0}$ is the space permeability, $R$ is the resistance of the electromagnet coil, and $N_{c}$ is the number of turns of the coil. The electro-magnetic force of a levitation unit is:

$$
F_{m}(t)=\frac{\mu_{0} A N_{c}^{2}}{2}\left(\frac{i(t)}{\delta(t)}\right)^{2}
$$

The vertical movement of the electromagnet can be described by:

$$
m_{1} \frac{\mathrm{d}^{2} y_{m}(t)}{\mathrm{d} t^{2}}=-F_{m}(t)+m_{1} g
$$

in which $m_{1}$ is the equivalent mass of the levitation payload. Detailed discussion of the stability of the electromagnet-track coupled system has been presented in 
previous published literature [3-5], which will not be repeated in this paper. Instead, as a demonstration, the stability of the coupled system discussed here will be investigated through numerical simulations. For example, if the parameters of the control system are chosen according to Table 2, and suppose that the track is well supported, the responses of the levitation gap and the displacement of the track can be obtained, which are shown in Fig. 7. It can be seen from Fig. 7(a) that, under the chosen parameters, the over-damped transient response of the levitation gap reflects a good performance of the levitation controller; more importantly, in this case, the vibratory response of the track tends to disappear, as shown in Fig. 7(b). This is a desired performance for a commercial maglev system.

Table 2. Parameters of the levitation control system

\begin{tabular}{|c|c|c|l|}
\hline Parameter & Value & Parameter & \multicolumn{1}{c|}{ Value } \\
\hline$k_{p}$ & 4000 & $m_{1}$ & $820 \mathrm{~kg}$ \\
\hline$k_{d}$ & 100 & $\mu_{0}$ & $4 \pi \times 10^{-7} \mathrm{H} / \mathrm{m}$ \\
\hline$k_{i}$ & 0.01 & $A$ & $0.01848 \mathrm{~m}^{2}$ \\
\hline$k_{c}$ & 100 & $N_{c}$ & 360 \\
\hline$l_{0}$ & $1.32 \mathrm{~m}$ & $R$ & $0.5 \mathrm{Ohm}$ \\
\hline
\end{tabular}
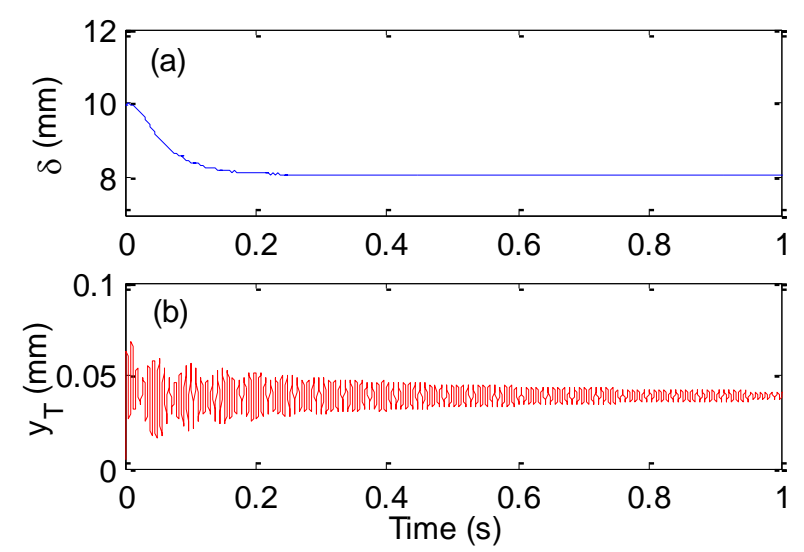

Fig. 7. Responses of the levitation gap and the displacement of the track under the well supported track

However, for the weakly supported track shown in Fig. 1, the fundamental frequency of the track drops dramatically, which greatly changes the stability of the electromagnet-track coupled system. Under the same parameters, the responses of the levitation gap and the track are shown in Fig. 8. Compared with Fig. 7, it is obvious that the electromagnet-track coupled system becomes unstable, and high frequency coupled resonance occurs. As the amplitude of the vibration grows, the noise arise which can be clearly heard by passengers aboard the train. This case has been encountered in the Tangshan low speed maglev test line, and it should be eliminated to prevent potential damage to track infrastructures. 


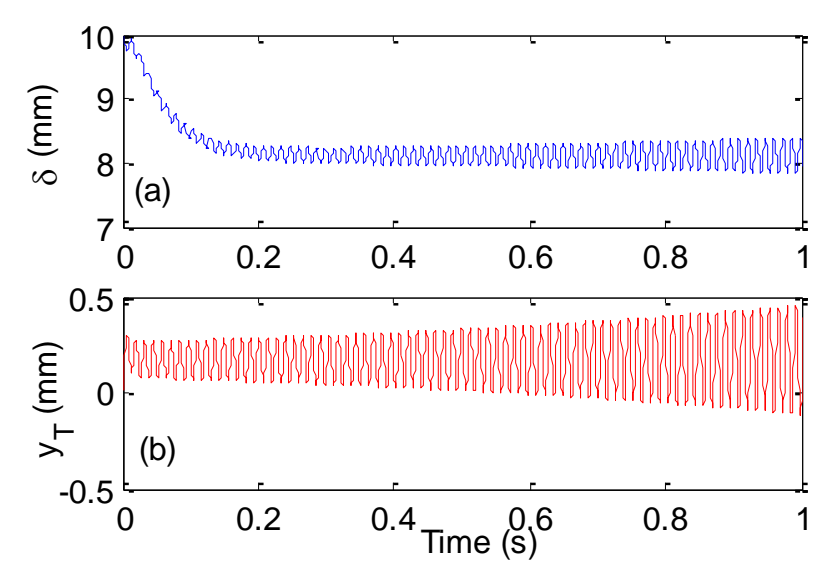

Fig. 8. Responses of the levitation gap and the displacement of the track under the weakly supported track

\section{ADAPTIVE VIBRATION CONTROL SCHEME FOR THE ELECTROMAGNET-TRACK COUPLED SYSTEM}

The self-excited vibration caused by weakly supported tracks seems inevitable for a practical maglev track since the fatigue or slack of screws may be unavoidable as a result of seasonal thermal expansions and contractions, as well as ground settlement. To eliminate the encountered self-excited vibration while the train is traveling, some special aspects should be taken into account: first, the frequency of the self-excited vibration is not a constant, but is variable due to the support conditions; second, the response of the vibration control scheme should be as rapid as possible; third, the vibration control scheme should not disturb the stability of the levitation controller, or at least it should not distinctly decrease the performance of the levitation control system.

Based on these considerations, an adaptive vibration control scheme, which is capable of tuning its parameters to cope with different track situations, is essential. Here, an adaptive vibration controller is proposed, as shown in Fig. 9. The block "CTL" represents the levitation controller which was described by Eqs. $(20-22)$, "MAG" is the electromagnet whose output includes the current and the electromagnetic force; " $\mathrm{H}(\mathrm{s})$ " is the transfer function of the track. The mechanism inside the dashed box is the designed adaptive vibration controller which includes two major parts: the frequency estimator which was designated by a block "FE", and the adaptive FIR filter with an adaptive tuning mechanism, "ADJ". The basic idea of this scheme can be generalized as follows: as the self-excited vibration occurs, the vibratory component mixed in the expected current, $i_{e 0}$, will exceed a certain value, and its frequency can then be estimated by the observer "FE"; a reference signal $x$, which is a sinusoid whose frequency equals the estimated frequency, $\omega_{r}$, is generated for the FIR queue, $\mathbf{X}$. The output of the FIR filter, $y_{f}$, is then subtracted from $i_{e 0}$, aiming to cancel the vibratory components in $i_{e 0}$. To achieve precise cancellation, the weights of the 
FIR filter, W, are adjusted by the adaptive mechanism ADJ, using the Least Mean Square (LMS) algorithm. If well tuned, the output of the cancellation, $i_{e}$, will not contain vibratory component, which indicates that the electromagnetic force, $F_{m}$, will not include vibratory excitation, either. Then, it can be inferred that the vibratory energy of the track will be dissipated as time elapses, and the self-excited vibration can thus be eliminated.

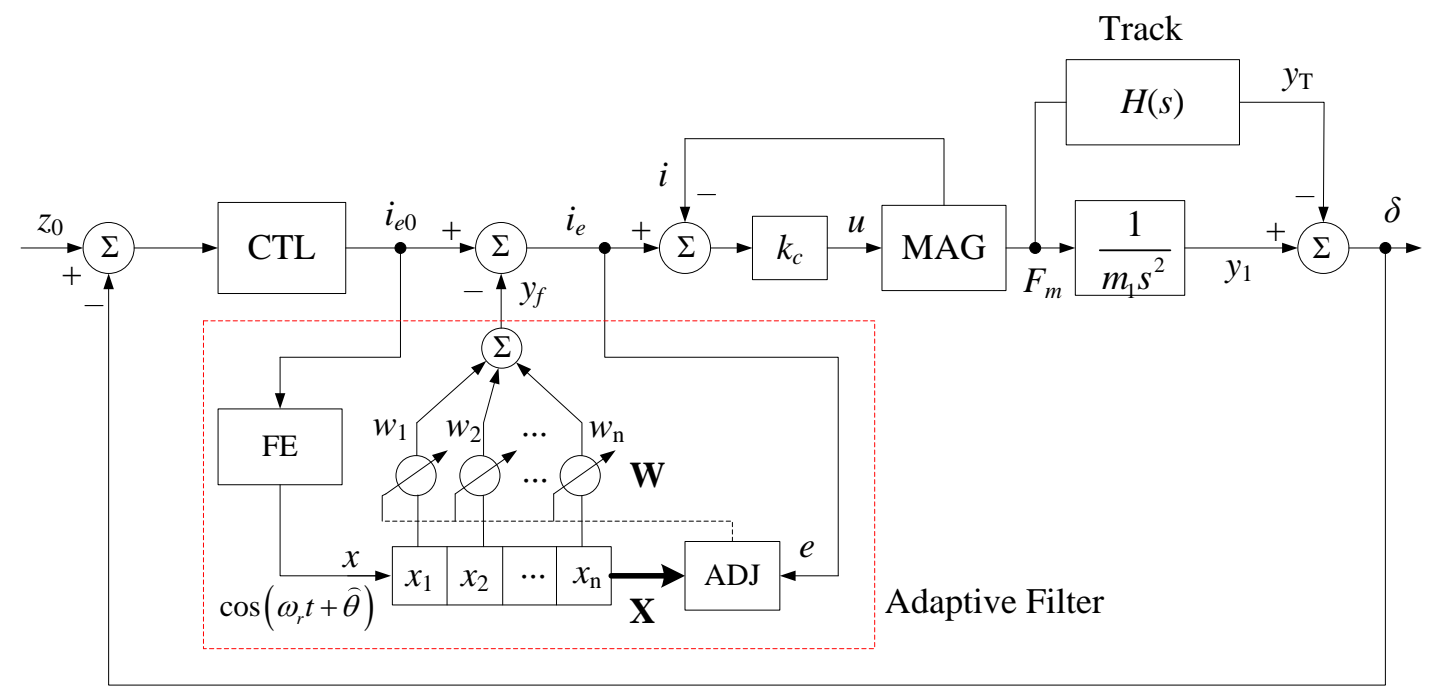

Fig. 9. Adaptive vibration control scheme combined with the maglev control system

From Fig. 9, it can be seen that

$$
\begin{aligned}
& y_{f}=\mathbf{W}^{T} \mathbf{X} \\
& i_{e}=i_{e 0}-y_{f}
\end{aligned}
$$

The purpose of the FIR filter is to produce a cancellation signal which is capable of eliminating the vibratory signal included in $i_{e 0}$, thus its weights needs to be tuned timely. The LMS algorithm is adopted here, and the weights of the FIR filter are updated according to the following rules:

$$
w_{n}(k+1)=\left(1-\alpha \mu_{1}\right) w_{n}(k)+2 \mu_{1} e x_{n}(k)
$$

where $\mu_{1}$ is the convergence coefficient of the LMS algorithm, $e$ is the cancellation error of the vibratory component in $i_{e}$, and $\alpha$ is a leakage coefficient that ameliorates the potential overflows of the weights caused by the accumulation of quantization errors.

To provide the FIR filter with a correct reference signal, a frequency estimator, FE, is utilized. Here, the frequency estimator developed by Regalia [9] is adopted here. This algorithm has been adopted by some other researchers, as in [10], which can be written as:

$$
\left\{\begin{array}{l}
\dot{x}_{1}=x_{2} \\
\dot{x}_{2}=-2 \zeta \omega_{r} x_{2}-\omega_{r}^{2} x_{1}+k u_{h f} \\
\dot{\omega}_{r}=-g\left(k u_{h f}-2 \zeta \omega_{r} x_{2}\right) x_{1}
\end{array}\right.
$$


where $u_{h f}$ is the input of the estimator. However, Fig. 9 is only a demonstration of the basic principle of the adaptive vibration controller. In practice, more considerations are required to keep the adaptive FIR filter work properly. It can be noted that $i_{e 0}$ contains not only the vibratory component which is associated to the vibration of the track, but also the DC (direct current) component which produces a biased current to levitate the payload; the latter, however, would cause the error signal for the LMS algorithm, e, superimposed with a DC component, which would prevent the LMS algorithm from converging. To solve this problem, high pass filters, as well as a compensation method introduced in [4], may also be applied here. For simplicity, it is not repeated in this paper.

Fig. 10 shows the test result of this adaptive vibration control method. The simulation is undertaken when all the parameters of the levitation system are chosen following the example in the previous section. For comparison, the adaptive vibration controller (AVC) was disabled for the first two seconds. As discussed earlier, due to the weak support of the track, self-excited vibration occurs, and the amplitude of the vibration grows with time. Fig. 10(b) shows the result of the frequency estimation, from which it can be seen that as the amplitude of the vibration exceeds a small value (corresponding to $0.9 \mathrm{~s}$ ), the frequency estimator begin to work and the result converges to the actual vibration frequency, which is $77 \mathrm{~Hz}$. Also, it can be seen from Fig. 10(c) that, the weights of the FIR filter are updated timely, and they are tuned in different manners as the reference frequency changes.
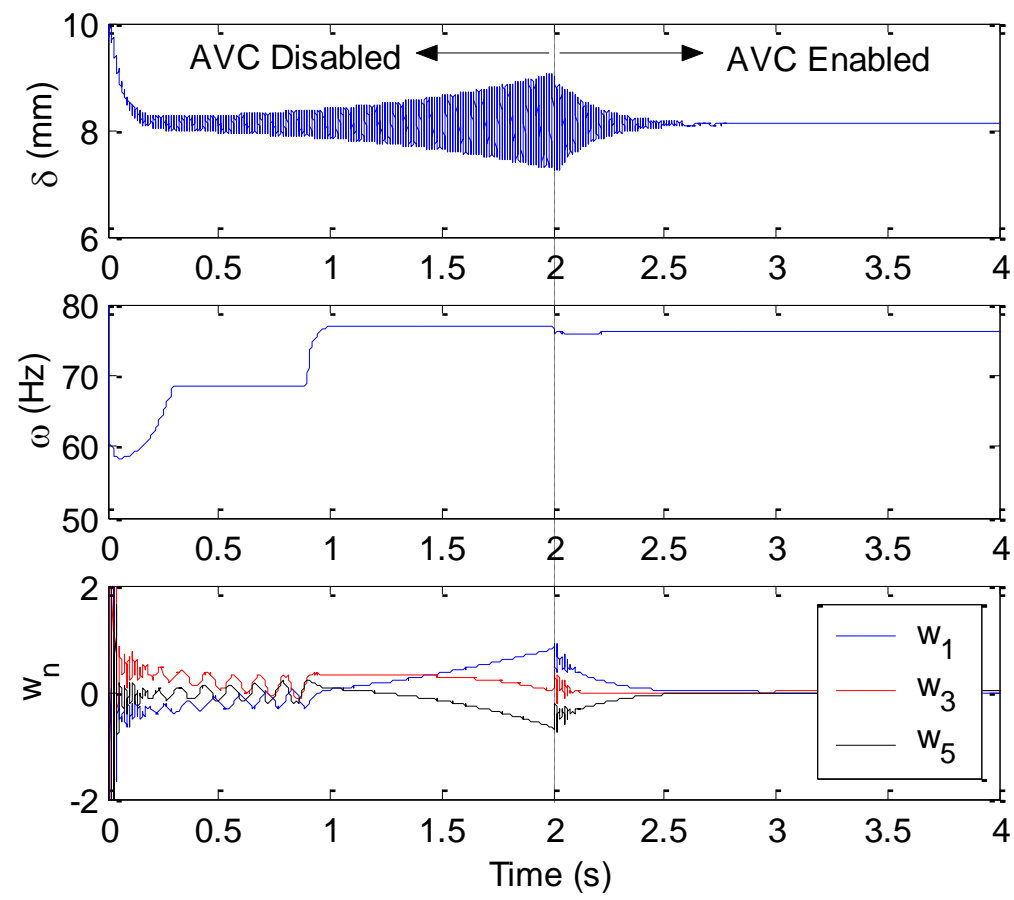

Fig. 10. Test result of the adaptive vibration control method. (a) the levitation gap; (b) estimated frequency; (c) selected weights of the adaptive FIR filter 
Then, when time equals $2 \mathrm{~s}$, the $\mathrm{AVC}$ is activated and the cancellation begins to take effect. It can be seen that the vibration begin to attenuate quickly afterwards. The weights of the adaptive filter also attenuate as the amplitude of the vibration decreases. The test result is satisfactory since the vibration of the result can be totally eliminated, and the electromagnet-track coupled system becomes stable.

\section{CONCLUSION}

In this paper, the principle of the track-induced coupled resonance problem in the urban maglev system is discussed. The major reason that lead to the electromagnet-track coupled vibration is analyzed, which reveals that the weak support of tracks may dramatically decrease the fundamental frequency of the track, whilst at the same time increase the mode gain of the fundamental vibration mode. These two factors destroy the conditions for the stability of the coupled system, leading to a self-excited vibration. Aiming to suppress the selfexcited vibration when a maglev train travels, an adaptive vibration controller, which includes a frequency estimator used to observe the frequency, and an adaptive FIR filter which produces a cancellation signal to neutralize the vibratory component mixed in the control path, is presented. The LMS algorithm is employed to tune the weights of the FIR filter, which achieves a satisfactory performance of convergence. The test result indicates that the proposed adaptive vibration controller is capable of suppressing the electromagnet-track coupled resonance.

\section{ACKNOWLEDGEMENT}

This work was supported by the National Key Development Program of the 13th Five-year plan [grant number 2016YFB1200601-B12]; the Research Plan Program of National University of Defense Technology [grant number 2K16-02-02]; the Opening Foundation of the State Key Laboratory of Functional Materials for Informatics [grant number SKL-2017-07]; and National Nature and Science Foundation of China [grant number 11302252].

\section{References}

1. Zhou DF, Hansen CH, Li J, Chang WS. Review of coupled vibration problems in EMS maglev vehicles. International Journal of Acoustics and Vibration. 2010;15(1):10-23. doi: 10.20855/ijav.2010.15.1255

2. Zhou DF, Hansen CH, Li J. Suppression of maglev vehicle-girder self-excited vibration using a virtual tuned mass damper. Journal of Sound and Vibration. 2011;330:883-901. doi:10.1016/j.jsv.2010.09.018

3. Zhou DF, Li J, Hansen $\mathrm{CH}$. Application of least mean square algorithm to suppression of maglev track-induced self-excited vibration. Journal of Sound and 
Vibration. 2011;330:5791-5811. doi: 10.1016/j.jsv.2011.07.021

4. Zhou DF, Li J, Zhang K. An Adaptive Control Method to Suppress the Maglev Track-Induced Self-Excited Vibration. Proceedings of the 2011 International Conference on Consumer Electronics, Communications and Networks; 2011; Xian Ning, China. p. 4723-4727. doi: 10.1109/CECNET.2011.5768485

5. Zhou DF, Li J, Zhang K. Amplitude control of the track-induced self-excited vibration for a maglev system. ISA Transactions. 2014; 53(5):1463-1469. doi: 10.1016/j.isatra.2013.12.016

6. Zhou DF. Research on Self-Excited Vibration Problems in EMS Maglev VehicleGuideway Coupled System [dissertation]. Graduate School of National University of Defense Technology; 2011 (in Chinese).

7. Svetlitsky VA. Engineering Vibration Analysis (Worked Problems 2). Berlin: Springer-Verlag; 2004. doi: 10.1007/978-3-540-40970-0

8. Zhou DF, Yu PC, Wang LC, Li J. An adaptive vibration control method to suppress the vibration of the maglev train caused by track irregularities. Journal of Sound and Vibration. 2017;408:331-350. doi: 10.1016/j.jsv.2017.07.037

9. Regalia PA. An improved lattice-based adaptive IIR notch filter. IEEE Trans. Signal Process. 1991;39(9):2124-2128. doi: 10.1109/78.134453

10. Bodson M, Douglas SC. Adaptive algorithms for the rejection of sinusoidal disturbances with unknown frequency. Automatica. 1997;33(12):2213-2221. doi: 10.1016/s0005-1098(97)00149-0

\section{Information about the authors:}

Danfeng Zhou, PhD, Lecturer; 109 Deya Road, National University of Defense Technology, Changsha, P.R. China.

ORCID: 0000-0001-5707-6530;

E-mail: zhoudanfeng@maglev.cn.

Peichang Yu, PhD, Lecturer;

E-mail: lofter@163.com.

Jie Li, PhD, Professor;

E-mail: 1j@maglev.cn.

Peng Cui, $\mathrm{PhD}$, Lecturer;

E-mail: cuipeng@maglev.cn.

Mengxiao Song, PhD, Student;

E-mail: songmengxiao14@nudt.edu.cn

To cite this article:

Zhou D, Yu P, Li J, et al. Adaptive Vibration Control of the Electromagnet-Track Coupled High Frequency Resonance for an Urban Maglev System. Transportation Systems and Technology. 2018;4(2):92-106. DOI: 10.17816/transsyst20184292-106 